\title{
Complex Regional Pain Syndrome Following Lumbar Discectomy
}

\author{
Do Hyun Kim, Hyung Sug Oh, Min Gi Kim, Tae Wan Kim, Kwan Ho Park \\ Department of Neurosurgery, VHS Medical Center, Seoul, Korea
}

\begin{abstract}
A 35-year-old man presented with back pain, radiating pain down to left leg. He had a past history of discectomy at L45 and L5S1 level. Left leg and foot pain was getting worse. We diagnosed herniated disc and performed discectomy at left L5S1level. He had had intractable pain, allodynia, and discolorization in left leg and foot. Lumbar magnetic resonance (MR) images showed post-laminectomy and epidural fibrosis. Three phase bone scan (TPBS) demonstrated increased blood flow and pooling at right leg and foot. Electromyography (EMG) study revealed left $L 5$ radiculopathy. The patient had undergone various types of procedures. The spinal cord stimulation (SCS) system was implanted. Left leg and foot pain was improved slightly. Intrathecal morphine pump (ITMP) was implanted. The physical examination showed hyperalgesia and allodynia in left leg and foot. In other hospital, SCS system was removed and dorsal root entry zone (DREZ) lesioning was performed. And then SCS implantation was performed at T89 level. CRPS was partially under control with pharmacotherapy and surgeries including SCS, ITMP, and DREZ lesioning. The authors report a case of CRPS following lumbar discectomy.
\end{abstract}

Key Words: Discectomy; Complex regional pain syndrome; Spinal cord stimulation; Intrathecal morphine pump; Dorsal root entry zone lesioning

$\triangle$ Corresponding Author: Kwan Ho Park, Department of Neurosurgery, VHS medical Center, 53 Jinwhangdoro 61 gil, Gangdong-gu, Seoul 05368, Korea. Tel: +82-2-2225-1363, Fax: +82-2-2225-4152, E-mail: spineho@naver.com

\section{INTRODUCTION}

Post-surgical spine syndrome (PSSS) refers to patients with persistent or new pain after spinal surgery. PSSS covers the pathological conditions that may be related to the surgery, such as nerve root compression or injury, epidural fibrosis, arachnoiditis, adjacent level degeneration, and spinal instability. Complex regional pain syndrome (CRPS) is a rare, chronic, and sometimes progressive condition. CRPS springs from trauma, nerve injury, operation, fracture, spinal cord injury and so on. The exact cause is unclear. CRPS has been reported following spinal surgery, including decompression/fusion, hemilaminectomy, microdiscectomy, and artificial disc replacement ${ }^{9,15,18)}$. The frequency of CPRS after spinal surgery is unknown ${ }^{17}$. CRPS requires an early diagnosis and successful treatment because if treatment is delayed, then patients will suffer from irreversible neurological changes of the affected limb.

We report a case of CPRS that was diagnosed with the causes of PSSS. A review of literature is discussed.

\section{CASE REPORT}

A 37-year old man was admitted for severe back pain, left leg pain and numbness radiating to the foot. It has happened again two years ago and worsening symptoms one month ago. He had a past history of discectomy at L45 and L5S1 level in other hospital. Lumbar magnetic resonance (MR) images showed post-laminectomy and herniated disc at left L5S1 level

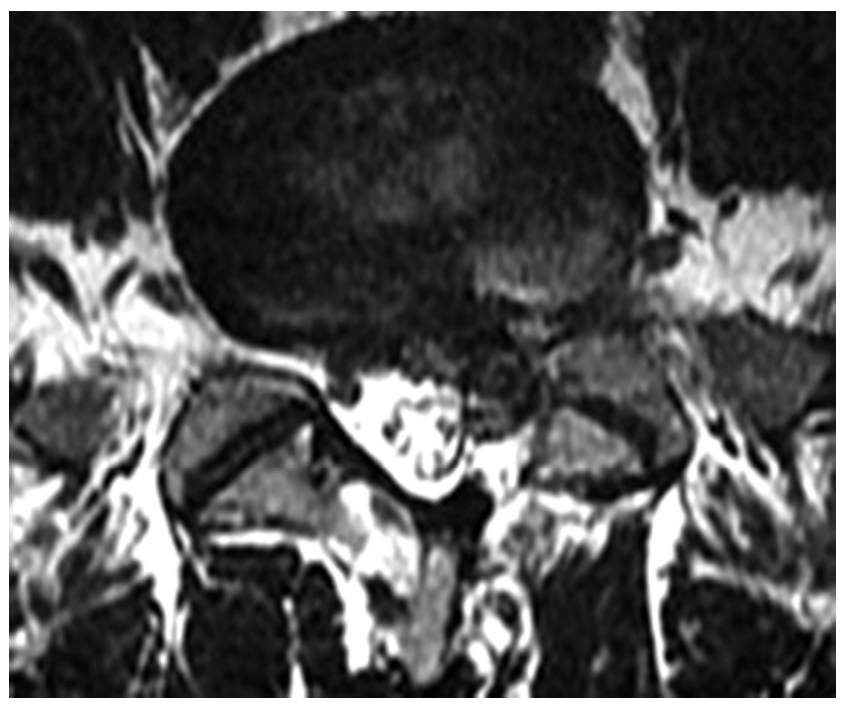

Fig. 1. T2 weighted axial MRI after previous surgery show epidural fibrosis and herniated disc at left L5S1. 
(Fig. 1). He underwent left-sided discectomy at L5S1 level. He experienced minimal improvement of the symptoms. He received various pain medications, physical therapy, and neural intervention therapies. His symptoms were slightly responsive to these treatments. The patient showed hyperesthesia, allodynia, discolorization, and dystrophic change in left leg and foot (Fig. 2). He was unable to walk because of intractable pain and tingling sensation.

Plain radiography showed no specific lesion. Angiography

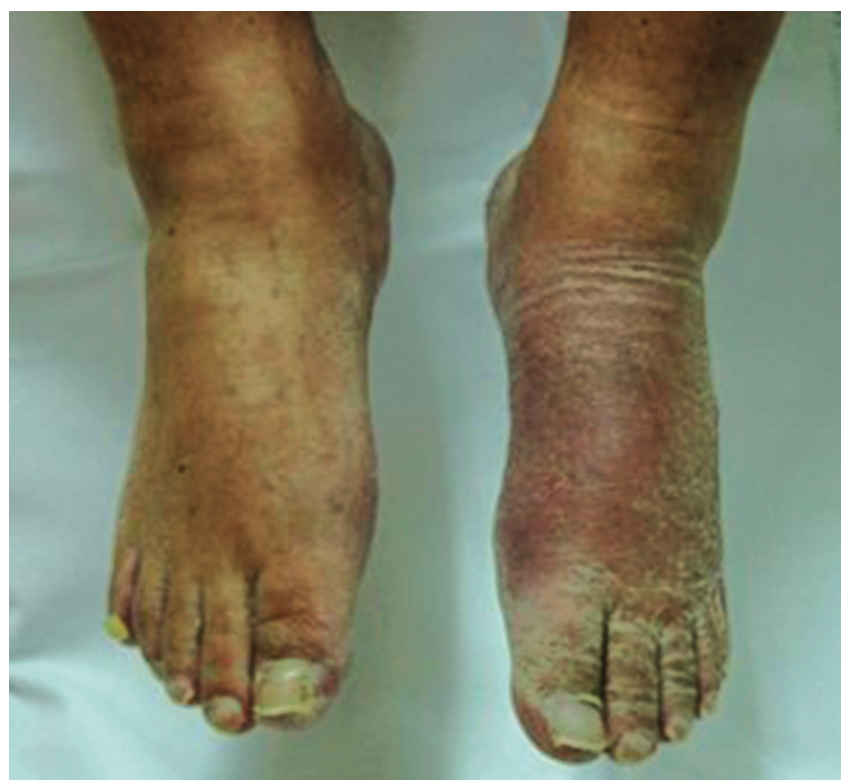

Fig. 2. Late CRPS of the left foot; red color and dystrophic skin.

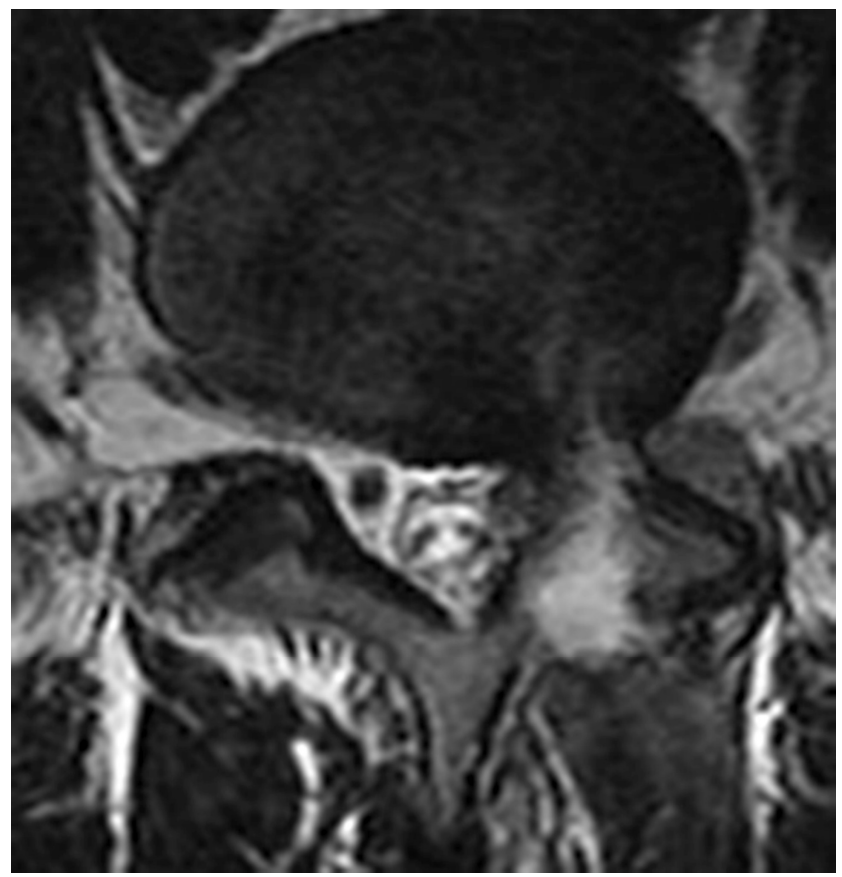

Fig. 3. T2 weighted axial MRI revealed no recurred disc and compressive lesion. revealed no evidence of vascular stenosis or obstruction. Lumbar MR images showed no recurred disc herniation and compressive lesion (Fig. 3). Three phase bone scan (TPBS) demonstrated increased blood flow and pool in right leg and foot (Fig. 4). We diagnosed the patients as having CRPS type 2 under the diagnostic criteria $^{8,16)}$

Pain medications and spinal block including sympathetic block were not effective. He underwent SCS implantation (Medtronic Inc, Minneapolis, MN, USA) between T10 and T12 (Fig. 5). There was some improvement of the pain in left leg and foot. The pain had deteriorated again. He suffered from intractable leg and foot pain. ITMP was implanted (Fig. 6). Intractable pain and allodynia remained in left leg and foot. He underwent surgery to remove percutaneous SCS system

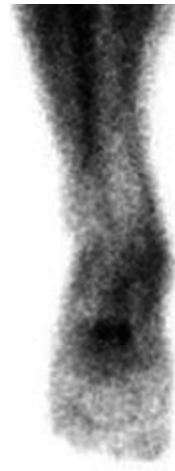

anterior

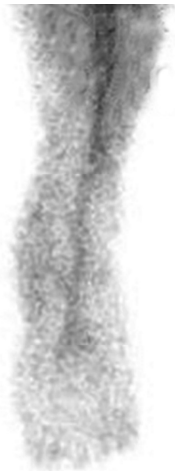

$3 \min$

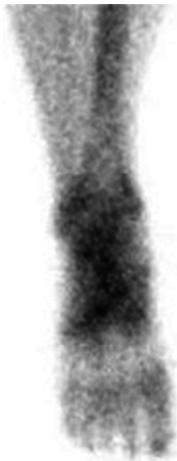

anterior

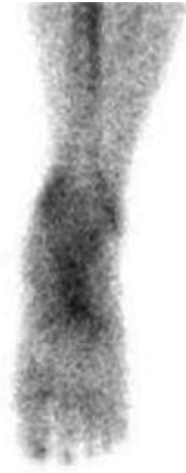

delay view
Fig. 4. Three phase bone scan demonstrated increased blood flow and pool in right leg and foot.

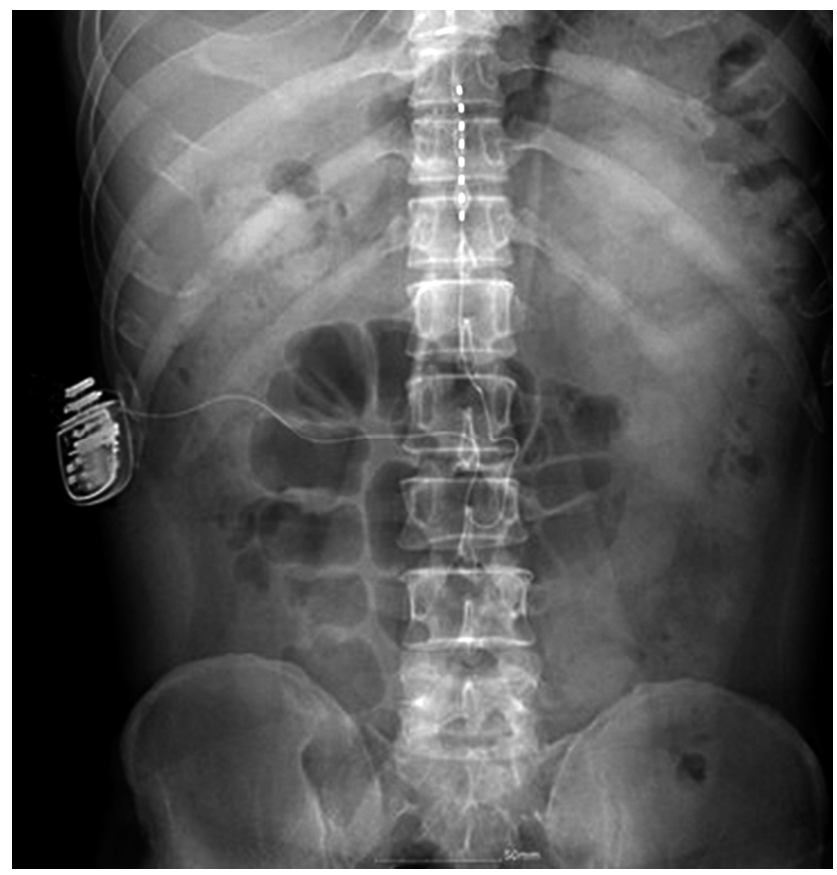

Fig. 5. Percutaneous lead was placed slightly off the midline between $\mathrm{T} 10$ and $\mathrm{T} 12$. 


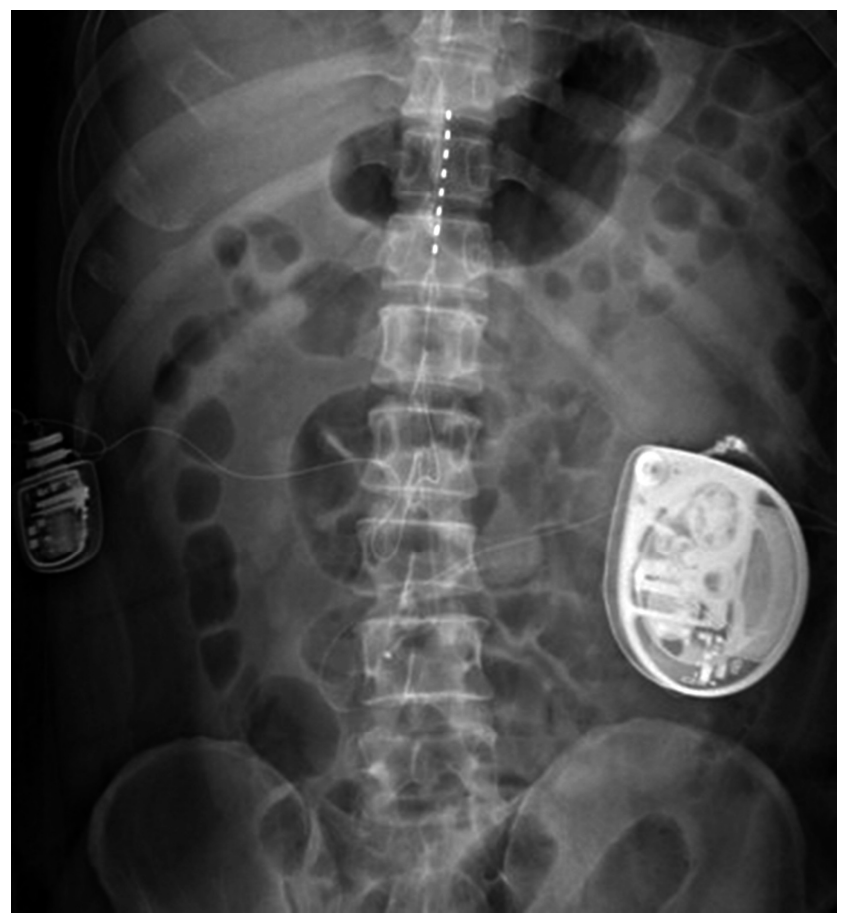

Fig. 6. Intrathecal morphine pump was implanted.

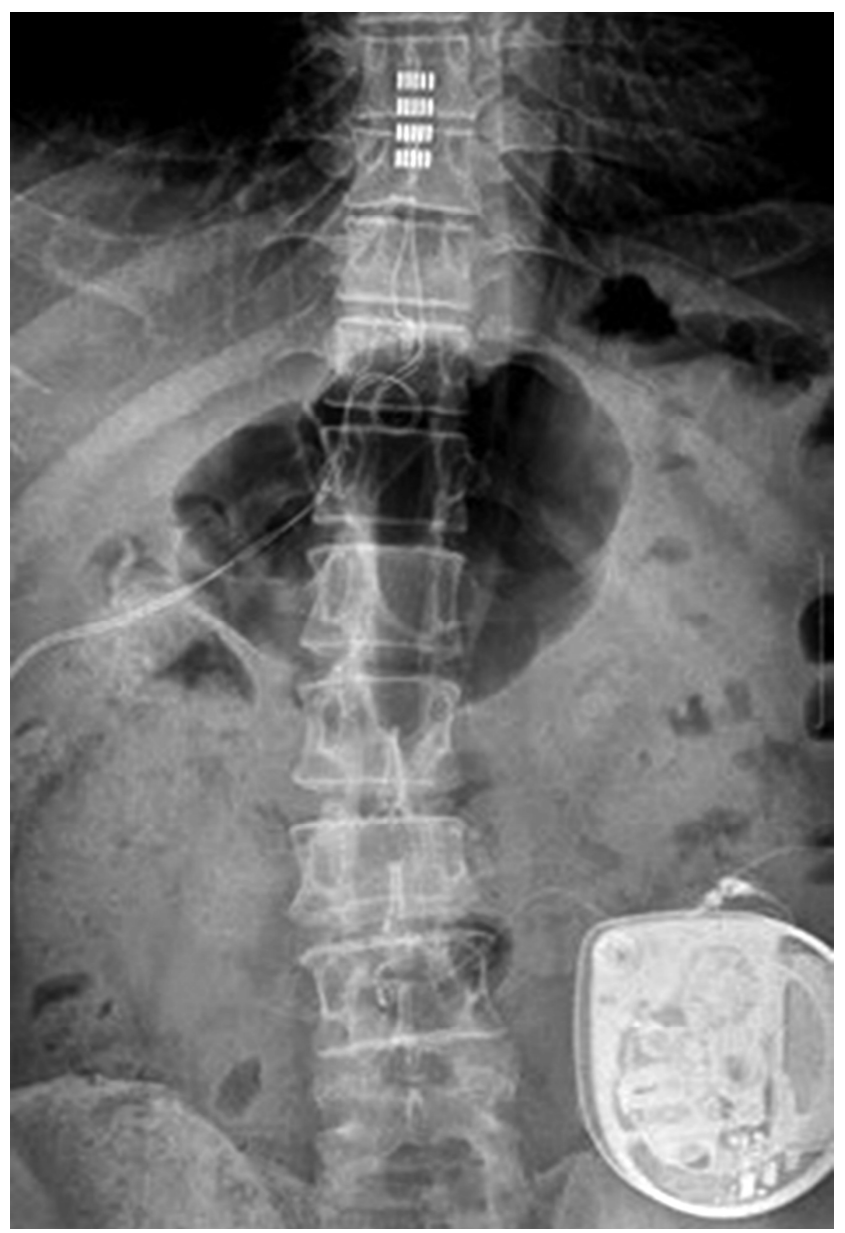

Fig. 7. A five-column Penta lead was implanted at T89 level. that did not work to reduce pain. Dorsal root entry zone (DREZ) lesioning at T12 segment was performed in other hospital. Sensation was decreased and mild weakness was developed in left leg and foot. There was restrictions on daily life and can't walk with extreme sole pain. And then SCS system (St Jude Medical, Inc., Saint Paul, MN, USA) implantation at T89 level was performed (Fig. 7). He had to receive pain medications. He suffered from walking difficulty because of the weakness and hyperalgesia and alloynia in left leg and foot.

\section{DISCUSSION}

As the number of cases of spinal surgery has increased, the cases of PSSS has also increased. There many causes of PSSS. Postoperative causes included infection, spinal instability, scar adhesion, adhesive arachnoiditis and problems of the fixation device after the operation. In this case, progressive neurogenic symptoms occurred and we assumed the causes of this symptoms were scar adhesion, adhesive arachnoiditis or a battered nerve root. Despite various treatments, the symptoms were aggravated, so we had a diagnosis of CRPS according to the diagnostic criteria.

Diagnosis of CRPS is based on clinical signs and symptoms according to the "Budapest criteria"; symptoms include pain, increased sensitivity, skin color, and temperature changes, as well as possible limb dysfunction ${ }^{4}$. The International Association for the Study of Pain (IASP) named and divided CRPS into type 1 and type 2 . Type 1 is similar to RSD without nerve injury after soft tissue injury, and type 2 is similar to causalgia after the nerve injury. This case belonged to CRPS type 2 . In 2004, IASP amended the diagnosis criteria of $\mathrm{CRPS}^{6}$.

CRPS is a neurogenic pain that is caused by trauma, fracture, operation, nerve injury etc. Typical CRPS symptoms includes autonomic, motor, and sensory abnormalities. It is characterized by various types of pain (tingling, allodynia, hyperalgesia etc), the functional loss of autonomous nerves and motor nerves, and by edema and dystrophy change. Theories of mechanism include antinflammatory process, a sympathetic nervous systemmediated disorder, central sensitization, cortical reorganization, autoimmune disease, limb ischemia with possible reperfusion injury, and nerve damage ${ }^{4)}$. CRPS is a rare complication after spine surgery. The emergence of CRPS II requires an additional impact on the nerve rather than mere nerve root compression. This impact might occur intraoperatively, in some cases it can also be due to nerve injury ${ }^{18)}$. The mechanism for the development of CRPS in this case could be related to damage to the nerve root during discectomy. CRPS occurs at a frequency of less than 1 in 2,000 patients following spine surgery ${ }^{18)}$. Knoeller et al. reported a case of CRPS following an anterior midline retroperitoneal approach for implantation of a lumbar artificial disc at the L45 level. They did not conclude sympathetic manipulation was the culprit ${ }^{9}$. Wolter et al. reported that CRPS 
symptoms developed in 5 patients after spine surgery ${ }^{18)}$.

In addition to a patient's symptom and physical examination, several diagnostic tests have been used to support diagnosis of CRPS. Clinical manifestation along with the use of imaging including plain radiography, TPBS and infrared thermography can help diagnose CRPS. However, the most important thing is the clinical decision with the exclusion of other diagnosis. Plain radiographs facilitate the diagnosis of CRPS in chronic stage as soon as bony changes develop. Demineralization with disuse and loss of function of the CRPS-affected area increases with time ${ }^{14)}$. The TPBS, which uses immediate and delayed images to study blood flow, is especially useful to CRPS study. Typically, CRPS shows increased blood flow, pool, and delayed periarticular uptake in affected limbs on TPBS $^{3)}$.

In this case, there was no typical finding on TPBS. There is still some controversy regarding the TPBS image criteria for CRPS. Although osteoporosis on plain radiograph or marrow edema and soft tissue swelling on MRI are supportive findings for CRPS-1, they are not as sensitive or specific as TPBS. MRI is helpful for eliminating differential diagnoses but no for diagnosing CRPS. Sympathetic block is injection of a local anesthetic to block the sympathetic nerves. The only way a doctor can find out if a CRPS patient's pain is sympatheticallymaintained pain (SMP), which is to do a sympathetic nerve block. There is no single proven diagnostic tool for CRPS diagnosis.

Therapy is based on an individual and multidisciplinary approach. Treatments include symptomatic treatment of pain, physical therapy, sympathetic block, SCS therapy, and ablation of neural structures. The efficacy of these treatments is debate and questionable. In this case, the patient did not improve with conservative treatment such as pain medications and various nerve blocks. Pain medications are a medical treatment option. Although some benefit was attained, pain relief was unable to be achieved with the medicine alone. Invasive pain treatment included sympathetic block, SCS, and ITMP. Sympathetic block was considered as a option for treating CRPS. The effect is still uncertain. Sympathetic block and another neural blocks were not effective in this case.

If noninvasive therapies fail, SCS represents an alternative therapeutic option. SCS was effective therapy for FBSS and CRPS, which is resistant to medical treatment, ${ }^{5,10,15)}$. SCS eliminates autonomic dysfunction, normalizes the micro-circulation and prevents allodynia and tingling. Calvillo et al. reported a series of 36 patients with advanced stages of CRPS (at least 2 years duration) who had undergone successful SCS therapy ${ }^{2}$. SCS appears to be an effective treatment option in patient with radiculopathy after lumbar disc surgery ${ }^{11)}$. The electrode of the spinal cord stimulator was placed on the appropriate part that induced paresthesia. A permanent spinal cord stimulator is generally implanted after a successful trial of stimulation. As time goes on, pain reduction was decreased. The patients experienced intractable pain. Although SCS is selected for chronic intractable pain treatment, the long-term outcome still remains controversial ${ }^{17}$. ITMP is used to provide strong pain relief by directly infusing low doses of morphine into the cerebrospinal fluid surrounding the opioid receptors. ITMP therapy is a useful option for patients with intractable CRPS who have failed other therapies, ${ }^{1,12)}$. The patient underwent ITMP implantation to treat intractable leg and foot pain. The pain was a little better but intractable pain and allodynia had persisted. The DREZ lesioning is a operation concerning the dorsal root entry region. DREZ lesioning is a destructive procedure and is associated with potential serious complications such as ataxia and limb weakness. DREZ?lesioning procedure may be a treatment option in selected cases of CRPS who are unresponsive to conventional therapies and surgical therapies, including nerve blocks, SCS and morphine pumps ${ }^{7}$. Prestor reported two cases who underwent DREZ coagulation for treatment of CRPS-I, with excellent results ${ }^{13}$. In other hospital, the patient underwent removal of percutaneous SCS system and DREZ lesioning operation at T12 segment. And then surgical SCS implantation at T89 level was performed. Despite all these treatments, the patient had been unsatisfactory in relieving the pain or in regaining limb functions and acceptable daily activities. We recommend suspecting CRPS as the cause of PSSS.

\section{CONCLUSION}

Although CRPS occurs rarely following spinal surgery, it is very important to be aware of the possibility that CRPS may occur. Differential diagnosis for CRPS at early stage is essential. Although the interventional therapies in CRPS may offer pain reduction, it is not expected to be effective.

\section{REFERENCES}

1. Becker WJ, Ablett DP, Harris CJ, Dold ON: Long term treatment of intractable reflex sympathetic dystrophy with intrathecal morphine. Can J Neurol Sci 22:153-159, 1995

2. Calvillo O, Racz G, Didie J, Smith K: Neuroaugmentation in the treatment of complex regional pain syndrome of the upper extremity. ActaOrthop Belg 64:57-63, 1998

3. Fournier RS, Holder LE: Reflex sympathetic dystrophy: diagnostic controversies. Semin Nucl Med 28:116-123, 1998

4. Goebel A: Complex regional pain syndrome in adults. Rheumatology 50:1739-1750, 2011

5. Gopal H, Fitzgerald J, McCrory C: Spinal cord stimulation for FBSS and CRPS: a review of 80 cases with on-table trial of stimulation. J Back Musculoskelet Rehabil 29:7-13, 2016

6. Harden RN, Bruehl S, Stanton-Hicks M, Wilson PR: Proposed new diagnostic criteria for complex regional pain syndrome. Pain Med 8:326-331, 2007

7. Kanpolat Y, Al-Beyati E, Ugur HC, Akpinar G, Kahilogullari G, Bozkurt M.A: Curative treatment option for complex regional pain syndrome (CRPS) Type I: dorsal root entry zone operation (report of two cases). Turk Neurosurg 24:127-130, 2014 
8. Kim YC: Complex regional pain syndrome in the Korean Pain Society (eds): Text book of Pain Medicine, ed2. Seoul: Koonja Publishing Inc, 2007, pp75

9. Knoeller SM, Ehmer M, Kleinmann B, Wolter T: CRPS I following artificial disc surgery: case report and review of the literature. Eur Spine J 20 (Suppl 2):S278-S283, 2011

10. Lee TK, Cho KS: Spinal neurostimulation for pain control: a review. Asian J Pain 1:30-35, 2015

11. North RB1, Kidd DH, Farrokhi F, Piantadosi SA: Spinal cord stimulation versus repeated lumbosacral spine surgery for chronic pain: a randomized, controlled trial. Neurosurgery 56:98-106, 2005

12. Oh HS, Oh HS, Park KH, Kim TW: Programmable intrathecal morphine pump. Asian J pain 1:24-29, 2015

13. Prestor B: Microsurgical junctional DREZ coagulation for treatment of deafferentation pain syndromes. Surg Neurol 56:259265, 2001

14. Rommel O, Häbler HJ, Schürmann M: Laboratory tests for com- plex regional pain syndrome in ilson P, Stanton-Hicks M, Harden RN (eds): CRPS: Current Diagnosis and Therapy. Progress in Pain Research and Management, Seattle: IASP Press, 2005, Vol 32, pp139-159

15. Sears NC, Machado AG, Nagel SJ, Deogaonkar M, Stanton-Hicks M, Rezai AR, et al.: Long-term outcomes of spinal cord stimulation with paddle leads in the treatment of complex regional pain syndrome and failed back surgery syndrome. Neuromodulation 14:312-318, 2011

16. Shin JW: Diagnosis and treatment of complex regional pain syndrome. Hanyang Med Review 31:76-84, 2011

17. Tsunetoh T, Moriyama K, Hirose M: Factors affecting outcome of spinal cord stimulation in pain associated with failed back surgery syndrome. J Pain Relief 4:5 2015. Available from: http:// dx.doi.org/ 10.4172/2167-0846.1000198

18. Wolter T, Knöller SM, Rommel O: Complex regional pain syndrome following spine surgery: clinical and prognostic implications. Eur Neurol 68:52-58, 2012 